\title{
Interactive Generation of Cities for Real-Time Applications
}

\author{
George Kelly* \\ Institute of Technology Blanchardstown, Ireland
}

\author{
Hugh McCabe M $^{\dagger}$ \\ Institute of Technology Blanchardstown, Ireland
}

This poster presents a design for an interactive city generation system for use in real-time applications. Our approach is to apply procedural techniques to allow the generation of a city and to enable features such as dynamic gaming environments and on-line distribution. An interactive application is outlined to manage the generation process from primary road creation to building construction. The focus of the system is on creating realistic large scale road networks and building models suitable for real-time rendering.

Previous research into the generation of cities has included Parish and Müller's CityEngine [2001], a system that combines geostatistical image maps and L-systems to create large scale road networks and detailed buildings suitable for cinematic rendering. Greuter et al. [2003] implemented a real-time solution that organises buildings along a regular grid enabling only the visible buildings to be generated and rendered. Wonka et al. [2003] have developed a formal grammar based on the concept of shape that can allow many detailed buildings to be generated.

The criteria used to evaluate a procedural generation solution are often the detail, complexity, heterogeneity and realism of the output. These indicators are crucial to the success of our system, but are not the only goals. To best solve the problem of generating cities suitable for real-time rendering a number of additional goals require consideration.

- Accessiblity - Input data such as geographical maps or geostatistical data should not be prerequisite to using the system.

- Interactivity - City generation can be fully autonomous but by allowing the option of user interaction the city can be tailored to specific requirements. The user can also control the patterns that the city is formed from on a local and global scale.

- Real-Time - Rendering considerations must be taken into account for real-time exploration of the city. A city model is a very large data set and techniques like selective culling, paging and level of detail should be implemented.

Our approach to achieving these goals is to design a system that is comprised of three major components: primary road generation, secondary road generation and building generation. These components will be integrated in a standalone application with all parameters and controls accessed through a visual interface providing an accessible workspace to perform city generation.

The primary road generation component utilises templates [Sun et al. 2002] that encapsulate common city road network patterns such as raster, radial, hierarchical and cellular. These templates are applied to a terrain in the form of an interconnected graph. The edges of the graph, the roads, can be automatically deformed by

\footnotetext{
*e-mail:george.kelly@itb.ie

†e-mail:hugh.mccabe@itb.ie
}

terrain characteristics such as gradients, water levels and other obstacles. The resultant road network graph is editable using an interactive 3D interface. Streets can be added, deleted and moved using junctions as control points for easy manipulation.

Cells created from the division of the city by the primary road network form units of area upon which the secondary road generation component can operate. A technique similar to that used in the CityEngine [2001] based on L-systems can be applied for secondary road generation. The algorithm will be applied within each cell to create roads that service the land area by providing access to and from the primary road network. Global and local parameters for each cell can control the operation of the secondary road generation. Our system aims to provide an environment in which these parameters are easily accessible and a live preview is available.

Buildings can be placed on the lots created by the enclosed regions from the secondary road generation. Building geometry will be constructed using L-systems taking several different building usage types into account including commercial, industrial, and residential with these usage types either stochastically assigned by the system or explicitly assigned by the user via the interactive interface.

Real-Time rendering is possible in our design via the provision of a number of optimization features: city cell paging, level of detail and real-time geometry generation. City cell paging is a concept similar to terrain paging systems, the primary road network forms a skeleton for the city containing the generation parameters for the entire city partitioned into cells. The secondary road networks within each cell are paged and can be pre-emptively generated and loaded on demand. Building structures stored as L-system strings can be parsed at run time to generate geometry. This provides a substantial reduction in memory usage by storing simple strings rather than complex geometry and also enables variants of buildings to be constructed depending on the parameters used for instantiation. A range dependent level of detail implementation can be constructed by including a parameter to specify the distance from camera to building and adjust the number of L-system iterations used accordingly.

The implementation of our design is currently in progress. The in teractive application has been built and the primary road generation component is complete, we expect to complete the final stages of the prototype in the next 6-8 months.

\section{References}

Greuter, S., Parker, J., Stewart, N., AND Leach, G. 2003. Real-time procedural generation of 'pseudo infinite' cities. In Proceedings of GRAPHITE 2003, ACM Press, 87-ff.

PARISh, Y. I. H., AND MÜller, P. 2001. Procedural modeling of cities. In SIGGRAPH 2001, Computer Graphics Proceedings, ACM Press / ACM SIGGRAPH, E. Fiume, Ed., 301-308.

Sun, J., Yu, X., Baciu, G., And Green, M. 2002. Templatebased generation of road networks for virtual city modeling. In VRST-02, ACM Press, H. Sun and Q. Peng, Eds., 33-40.

Wonka, P., Wimmer, M., Sillion, F., AND RibARSKY, W. 2003. Instant architecture. In Proceedings of ACM SIGGRAPH 2003, J. Hodgins and J. C. Hart, Eds., vol. 22(3), 669-677. 